\title{
LAS UNIDADES MÍNIMAS DEL DISCURSO Y LAS ESTRUCTURAS MÁS FRECUENTES EN LA CONVERSACIÓN COLOQUIAL
}

\author{
THE SMALLEST DISCOURSE UNITS AND THE MOST FREQUENT STRUCTURES IN \\ COLLOQUIAL CONVERSATION
}

\section{Lidia Latorre García}

Universitat de València

Resumen

El objetivo principal de este artículo es estudiar la distribución de la información en la conversación coloquial en español a partir del análisis de la unidad mínima subacto (Briz y Grupo Val.Es.Co., 2003, Grupo Val.Es.Co., 2014). El subacto es la unidad relacionada con la dimensión informativa del discurso y diferencia contenidos proposicionales y procedimentales. En esta investigación se han segmentado un total 621 actos y 1188 subactos pertenecientes a conversaciones coloquiales. Seguidamente, se han contabilizado los patrones de aparición más frecuentes y se ha examinado la relación existente entre los diferentes tipos de subactos. Los resultados de este estudio muestran que los actos simples conforman el patrón más frecuente en la conversación coloquial española y que los actos tienden a ser introducidos por un contenido procedimental.

Palabras Clave: unidades del discurso, subacto, estructura informativa, conversación coloquial.
Abstract

The goal of this paper is to study the distribution of linguistic information in colloquial Spanish conversation, by analysing the smallest discourse unit: the subact (Briz y Grupo Val.Es.Co., 2003, Grupo Val.Es.Co., 2014). Subacts deal with the distribution of information and distinguish between propositional and procedural contents. In this study, three spontaneous conversations were segmented into a total of 621 acts and 1188 subacts. The most frequent patterns of occurrence have been extracted and the relationship between the different types of subacts was analysed. The results show that single acts are the most frequent pattern in colloquial Spanish conversation and that acts are usually started with procedural content.

KEY WORDS: discourse units, subact, information structure, colloquial conversation 


\section{INTRODUCCIÓN}

\subsection{Los sistemas de segmentación del discurso oral}

La consolidación de los sistemas de segmentación del discurso oral como herramienta de análisis se produjo a partir del tercer cuarto del siglo XX. Es en esta época cuando, sobre todo en el ámbito románico, crece el interés por el estudio de fenómenos orales que la sintaxis descriptiva no podía abarcar: por ejemplo, la noción de oración, o la clasificación de oraciones en coordinadas y subordinadas (especialmente el caso de las subordinadas adverbiales impropias) requerían un tratamiento especial en los discursos orales. Entre todos, el discurso oral coloquial era el que más dificultades presentaba: las herramientas de análisis de la sintaxis descriptiva tradicional no abordaban estos fenómenos sin caer en explicaciones que acabaran dejándolos a un lado o catalogándolos como problemas por su falta de similitud con lo escrito (Narbona, 1989a, 1989b, 1990, Briz y Grupo Val.Es.Co., 2003). Por esta razón surgió la necesidad de «llevar a cabo una descripción global rigurosa de los tipos de esquema constructivos dominantes en la lengua coloquial y de la técnica a la que responden» (Narbona, 1994: 724).

Esta descripción de la lengua coloquial y su funcionamiento fue desarrollada por varios grupos de investigación en el ámbito románico desde la década de los años noventa. La propuesta de tratamiento que estos grupos ofrecieron consistió en la creación de modelos de segmentación discursiva que, sin copiar las mismas unidades de análisis de la sintaxis tradicional, pudieran dar cuenta de los diferentes fenómenos lingüísticos desde una perspectiva estructural, formal y/o funcional (Roulet et alii, 1985, Blanche-Benveniste, 1990, Roulet, 1991, Morel y Danon-Boileau, 1998, Cortés, 2002, Cortés y Camacho, 2005, Briz y Grupo Val.Es.Co., 2003, Degand y Simon, 2005, 2008, 2009, Berrendonner, 2011, Grupo Val.Es.Co., 2014, Pons Bordería, 2014). La segmentación en unidades y subunidades, además, permitía analizar cualitativa y cuantitativamente dichos fenómenos.

Los modelos desarrollados por estos grupos proponen una serie cerrada de unidades discursivas que atienden a diferentes criterios, los cuales determinan el tipo y la cantidad de unidades disponibles para el analista. Estos criterios son:

a) El criterio prosódico, que parte de la entonación y de los índices suprasegmentales para delimitar las unidades en las que se segmenta una conversación. Está presente en el Modelo de Co-enunciación (Morel y Danon-Boileau, 1998) y en el del Grupo de Friburgo (Berrendonner, 2011).

b) El criterio discursivo, que delimita las unidades según el progreso en la estructura discursiva y, por tanto, los cambios en la memoria discursiva. Este criterio constituye la base del modelo desarrollado por Roulet (1985, Roulet et alii, 1991).

c) Los criterios prosódicos y discursivos combinados dan lugar a modelos de unidades polivalentes. Algunas de las combinaciones más frecuentes consideran que la sintaxis y la prosodia pueden complementarse para dar lugar a unidades estructurales que permiten dobles análisis. Este es el caso del modelo desarrollado por Blanche-Benveniste (1990) y el de Degand y Simon (2005, 2008, 2009). Otras combinaciones aúnan la prosodia, el contenido proposicional y la fuerza ilocutiva para generar análisis conversacionales tridimensionales. Es el caso del modelo desarrollado por el grupo Val.Es.Co. (Briz y Grupo Val.Es.Co., 2003, Grupo Val.Es.Co., 2014). 
La presente investigación toma como referencia las unidades propuestas por este último grupo. Asimismo, selecciona como objeto de estudio las unidades mínimas por su condición de frontera entre la sintaxis oracional y el análisis del discurso. Debido a esta circunstancia, las unidades mínimas son un campo de estudio productivo que está siendo explotado en diferentes artículos y proyectos de investigación durante los últimos años (Hidalgo y Padilla, 2006, Padilla, 2006, Cabedo, 2009, Pascual Aliaga, 2014, Pons Bordería, 2016). A continuación, se exponen las diferentes unidades que componen este modelo jerárquico.

\title{
1.2 El modelo del Grupo Val.Es.Co.
}

El modelo del Grupo Val.Es.Co. es jerárquico y recursivo y está formado por tres dimensiones (estructural, social e informativa) y ocho unidades (discurso, diálogo, intercambio, alternancia de turnos, intervención, turno, acto y subacto) analizables en dos niveles (monológico y diálogico) (Grupo Val.Es.Co., 2014).

Las unidades que interesan a esta investigación son el acto y el subacto ${ }^{1}$. Así, el acto es definido como:

\begin{abstract}
Unidad estructural monológica, jerárquicamente inferior a la intervención, de la que es su constituyente inmediato. Todo acto se constituye primariamente como la unión de una forma lógica o proposicional más una serie de subactos adyacentes asociados a la misma. Este criterio se matiza secundariamente con la adición de dos criterios: prosódico (presencia de un grupo entonativo completo), y pragmático (posesión de fuerza ilocutiva propia). En su ámbito prototípico (la conversación coloquial), el acto prototípico posee las propiedades de aislabilidad e independencia (Pons Bordería, 2016: 556).
\end{abstract}

La aislabilidad se relaciona con el hecho de poseer fuerza ilocutiva propia, y la identificabilidad con la presencia de algunas marcas lingüísticas, prosódicas y semánticas que la delimitan².

El subacto es la «unidad monológica estructural, constituyente inmediata del acto, caracterizada por constituir un segmento informativo identificable, habitualmente, mediante marcas semánticas y prosódicas» (Grupo Val.Es.Co., 2014: 53).

Es un segmento informativo en el sentido de que expresa diferentes valores de significado como causa o condición; es en este aspecto en el que un acto sí puede dividirse. Los subactos se clasifican en dos grandes grupos: los sustantivos si poseen contenido proposicional, y los adyacentes si no lo poseen. Ambas clases se subdividen en tres tipos:

- Subacto sustantivo director (SSD): es el portador de la fuerza ilocutiva del acto, por lo que es el subacto mínimo necesario para la constitución de un acto.

- Subacto sustantivo subordinado (SSS): depende semántica e informativamente del SSD al que acompaña y aporta «informaciones secundarias (causa, concesión, consecuencia, finalidad, tiempo, lugar, topicalización, etc.)» (Grupo Val.Es.Co., 2014: 59).

- Subacto sustantivo subordinado topicalizado (SSTop): «una topicalización es un cambio de posición de un constituyente oracional (y/o proposicional) a una posición

\footnotetext{
${ }_{1}$ Para un tratamiento exhaustivo de las diferentes unidades que componen el modelo, véase Grupo Val.Es.Co., 2014.

2 Algunas de estas marcas son la presencia de una curva melódica completa o la posibilidad de ser sustituidos por otros actos como prueba de su independencia (Grupo Val.Es.Co., 2014: 37-47). 
informativamente relevante (primera o última)» (Grupo Val.Es.Co., 2014: 60-61). Son subordinados debido a que su carga informativa está supeditada a un SSD.

- Subacto adyacente textual (SAT): es el encargado de organizar y distribuir el flujo de habla. Algunos marcadores discursivos como entonces desempeñan esta función.

- Subacto adyacente interpersonal (SAI): comprende la interacción entre los interlocutores. Prototípicamente, esta función se realiza a través de los marcadores discursivos de control del contacto como ¿no?

- Subacto adyacente modalizador (SAM): incluye matizaciones modales concretas (atenuación, intensificación) en el subacto sustantivo al que se adhiere. Las expresiones que pueden desempeñar este papel son del tipo digo yo o yo qué sé.

Por último, existen subactos que, por su naturaleza semántico-informativa ambigua, se han clasificado bajo las siguientes etiquetas: SXX (subacto de naturaleza indeterminada), SSX (subacto sustantivo de naturaleza indeterminada) y SAX (subacto adyacente de naturaleza indeterminada) (Pons Bordería, 2016: 560)³.

\subsection{Planteamiento y objetivos}

De las definiciones anteriores se deduce que el subacto se sitúa en la frontera entre la sintaxis descriptiva y el análisis del discurso, lo que permite abordar fenómenos muy cercanos a estructuras propias de la sintaxis tradicional (subordinación, coordinación, estructuración y organización de contenidos, etc.) pero desde una visión discursiva (fenómenos entre el decir y lo dicho, coordinación y organización de contenidos propios de una planificación del discurso reducida, marcación del discurso en diferentes niveles, etc.). El subacto incorpora la dimensión informativa del modelo, por lo que su estudio puede contribuir a la investigación sobre la estructura de la información.

Este estudio tiene como objetivos principales tres puntos clave:

a) estudiar cómo se construyen las intervenciones en la conversación coloquial en español,

b) extraer las estructuras de aparición más frecuente en la conversación coloquial,

c) analizar la ordenación de la información en estas intervenciones.

\section{Metodología}

\subsection{Delimitación del corpus}

Para esta investigación se han segmentado y analizado los primeros 207 actos de las conversaciones 42, 43 y 44 del Corpus Val.Es.Co. 2.0. (Cabedo y Pons Bordería, 2013) con la ayuda del programa ELAN ${ }^{4}$. Para la segmentación se han seguido las pautas de

\footnotetext{
${ }^{3}$ El uso de estas etiquetas permite un acercamiento detallado a fenómenos comunicativos que se encuentran a la vez en territorios funcionales diferentes: marcadores discursivos que cubren dos funciones simultáneamente, elementos léxicos que presentan marcas procedimentales, o fenómenos suprasegmentales como los reinicios o vacilaciones (Pascual Aliaga, tesis en progreso, comunicación personal).

${ }^{4}$ ELAN es un programa de transcripción multimodal que permite realizar anotaciones en líneas digitales de información, para distintos archivos de audio y vídeo (Sloetjes y Wittenburg, 2008). 
análisis propuestas en Pons Bordería (2016: 557-558). Las unidades han sido analizadas en el siguiente orden:

- En primer lugar, se señalan los subactos adyacentes (SSAA) y se les asigna una función.

- En segundo lugar, se señalan los elementos entre los SSAA como subactos sustantivos y se les asigna una función.

- En tercer lugar, se considera como acto la unión entre los contenidos proposicionales (subactos sustantivos) y los contenidos no proposicionales o procedimentales (subactos adyacentes).

Si bien las segmentaciones de la investigación son de la autora, el conjunto del corpus se ha contrastado y comparado con segmentaciones realizadas por diferentes miembros del Grupo Val.Es.Co. (Pascual Aliaga, 2014, entre otros).

El siguiente fragmento reproduce el orden de segmentación seguido:

(1) C: ipues igual que tú! // ((ija!)) // jpiltrafilla!

A: como dice el de Jerez // cuando le piden el deneí porque tiene un- cara de ((nene)) [(( bueno ahora- )) ahora ya] no pero co- con venti- ahora tiene veintii- ((uuh)) ahora tiene vein tii ssiete para veintiocho ¿eh?

El ejemplo (1) presenta dos intervenciones: una primera, del hablante C, y una segunda, más extensa, emitida por el hablante A. Primero se señalan los SSAA, es decir, aquellos que poseen el contenido procedimental, y se les asigna una función. Para su demarcación se utilizan corchetes y las siglas del tipo de subacto que encierran, como se extrae de (2):

(2) C: i\{SAT pues SAT $\}$ igual que tú! // \{SAM ((ija!)) SAM $\} / /$ i\{SAl piltrafilla! SAl\}

A: como dice el de Jerez // cuando le piden el deneí porque tiene un- cara de ((nene)) [( \{SAT bueno SAT\} ahora- )) ahora ya] no pero co- con venti- ahora tiene veintii- ((uuh)) ahora tiene vein tii ssiete para veintiocho $\{$ SAl ¿eh? SAl $\}$

A continuación, se señalan los elementos que se encuentran entre los subactos adyacentes como subactos sustantivos y se les asigna una función atendiendo a los tres criterios ya mencionados. El resultado es el que se muestra en (3):

(3) C: ¡\{SAT pues SAT\} \{SSD igual que tú! SSD\} // \{SAM ((ija!)) SAM\} // iSSAI piltrafilla! SAI\}

A: \{SSS como dice el de Jerez SSS\} // \{SSS cuando le piden el deneí porque tiene un- cara de ((nene)) SSS\} [( \{SAT bueno SAT\} \{SSD ahora- )) ahora ya] no pero co- con venti- ahora tiene veintii- ((uuh)) ahora tiene vein tii ssiete para veintiocho SSD\} \{SAl ¿eh? SAl\}

Por último, se delimitan los actos con su marca correspondiente (\#). (4) es el resultado final de la segmentación:

(4) C: \# i\{SAT pues SAT\} \{SSD igual que tú! SSD $/ /$ \{SAM ((ija!)) SAM $\} / /$ i\{SAl piltrafilla! SAI\} \# A: \# \{SSS como dice el de Jerez SSS\} // \{SSS cuando le piden el deneí porque tiene un- cara de ((nene)) SSS\} [( \{SAT bueno SAT\} \{SSD ahora- )) ahora ya] no pero co- con venti- ahora tiene veintii- ((uuh)) ahora tiene vein tii ssiete para veintiocho SSD $\{$ SAI ¿eh? SAl\} \#

Esta segmentación sigue los tres criterios expuestos anteriormente: proposicional, ilocutivo y prosódico. Dado que no en todos los casos los tres criterios delimitaban las unidades de igual manera, se ha realizado la siguiente priorización:

a) Cuando el criterio prosódico no coincide con el ilocutivo y el proposicional, se tienen en cuenta estos dos últimos, ya que es frecuente que los subactos adyacentes no se 
correspondan con grupos entonativos, pero sí cumplan los criterios ilocutivo, proposicional e informativo. Además, la planificación sobre la marcha de la conversación coloquial hace que en numerosas ocasiones los hablantes hagan pausas o modifiquen su discurso a medida que lo producen, creando así varios grupos entonativos que representan únicamente un contenido informativo y una intención.

b) Cuando el criterio proposicional no coincide con el ilocutivo, se prioriza este último, es decir, la intención única. Esta decisión se ve justificada por el hecho de que el hablante puede cambiar la forma en la que expresa su intención a medida que construye su discurso, pero el aporte al funcionamiento de la conversación es el mismo.

c) En los fragmentos con estilo directo se sigue una segmentación distinta, por las características concretas de este tipo de discurso5. En estas segmentaciones se señala como SAT el verbo o construcción que introduce el discurso directo dentro del acto donde se encuentra este mismo discurso, como se muestra en el ejemplo (5):

(5) B: \#\{SSD porque Dani ((Olucha)) es igual de malo que él SSD\} \{SAT [peroo] SAT\}\#

A: \# \{SSD [pero] pasa SSD $\}$ \#

B: \# $\{\{S S D$ le pones a correr SSD $\}$ \# \# \{SAT y dice SAT $\}$ / / [SSSD [no yo de defensa] SSD $\}$ \#

\subsection{Análisis del corpus}

Tras segmentar todas las conversaciones, se crea una base de datos. Esta base codifica diferentes tipos de información:

a) El número de identificación.

b) La conversación de la que procede el segmento.

c) El emisor: lo que permite, en un análisis preliminar, descartar que los resultados obtenidos son idiosincrásicos de un hablante o hablantes concretos.

d) El acto estudiado con los subactos que lo componen: es el segmento lingüístico analizado y del que se extrae toda la información.

e) El esquema de subactos: permite establecer patrones de (co)aparición y resulta necesario para el análisis cuantitativo.

f) El número de subactos: del mismo modo que el esquema, se utiliza para realizar las operaciones estadísticas y encontrar frecuencias de aparición.

g) Estilo directo: señalar la presencia de estilo directo posibilita un análisis que incluya o excluya estos casos.

Posteriormente, se procede a la extracción de frecuencias de aparición: de los esquemas de subactos, de los esquemas de subactos «estilizados» (agrupando los diferentes tipos de subactos adyacentes por un lado y los sustantivos por otro) y de los subactos que aparecen en primera posición de acto.

Para asegurar que los datos obtenidos no se deben a la influencia del azar, se han aplicado pruebas estadísticas chi cuadrado. Este test permite conocer la probabilidad de obtener

${ }_{5}^{5}$ Para una explicación más completa de este aspecto, véase Benavent Payá (2015). 
unos resultados similares si esta investigación se replicara en futuros trabajos. La prueba se ha aplicado a los patrones de aparición y a los patrones de orden manifestados por los subactos en el corpus segmentado.

\section{ANÁLISIS Y RESULTADOS}

\subsection{Frecuencias de aparición}

El primer objetivo de esta investigación es estudiar el modo en el que se construyen las intervenciones de las conversaciones coloquiales españolas. En primer lugar, se analiza cómo son los actos que componen las intervenciones, de manera cuantitativa, clasificándolos por el número de subactos de los que constan. El primer gráfico muestra la distribución de número de actos según el número de subactos que los componen:

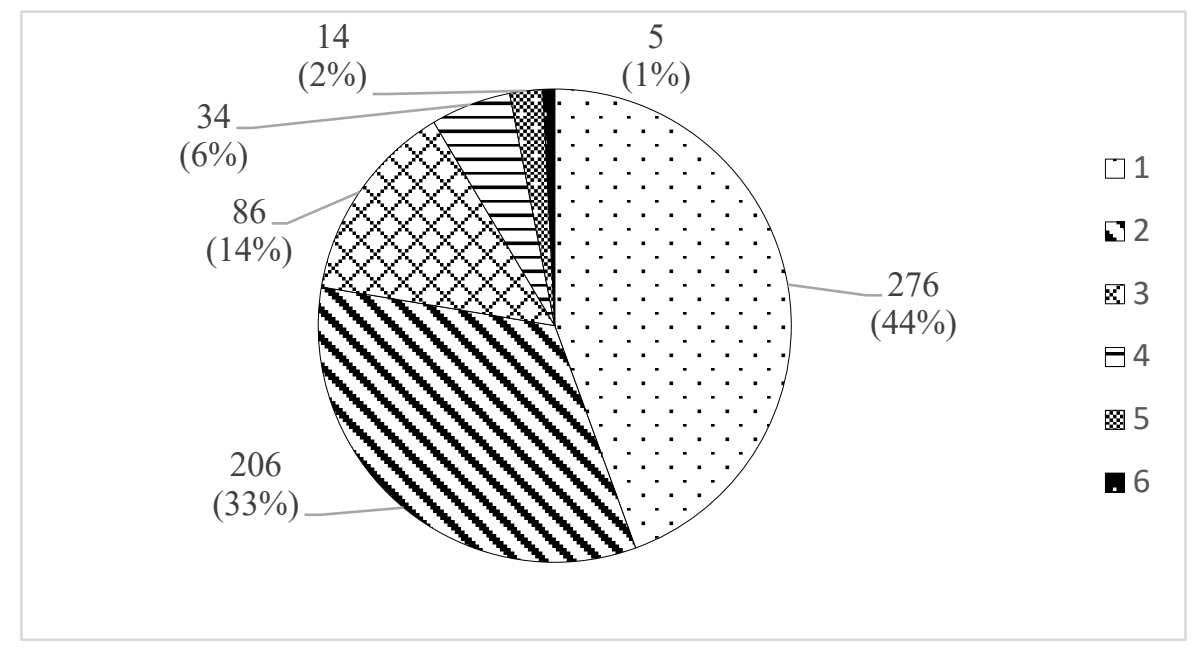

Gráfico 1: Distribución de actos por número de subactos

El $77 \%$ de los actos del corpus de esta investigación están compuestos por uno o dos subactos. Esta cifra señala la simplicidad estructural de la mayoría de actos frente a la tendencia de entender la conversación coloquial como un fenómeno de complicado análisis. Estos datos resultan una muestra de cómo un modelo de segmentación facilita el estudio y análisis de fenómenos o elementos lingüísticos señalados como problemáticos.

El segundo objetivo del estudio consiste en extraer las estructuras de aparición más habitual. Para ello, se han analizado los patrones más frecuentes en los distintos actos a partir del número de subactos que los componen: 


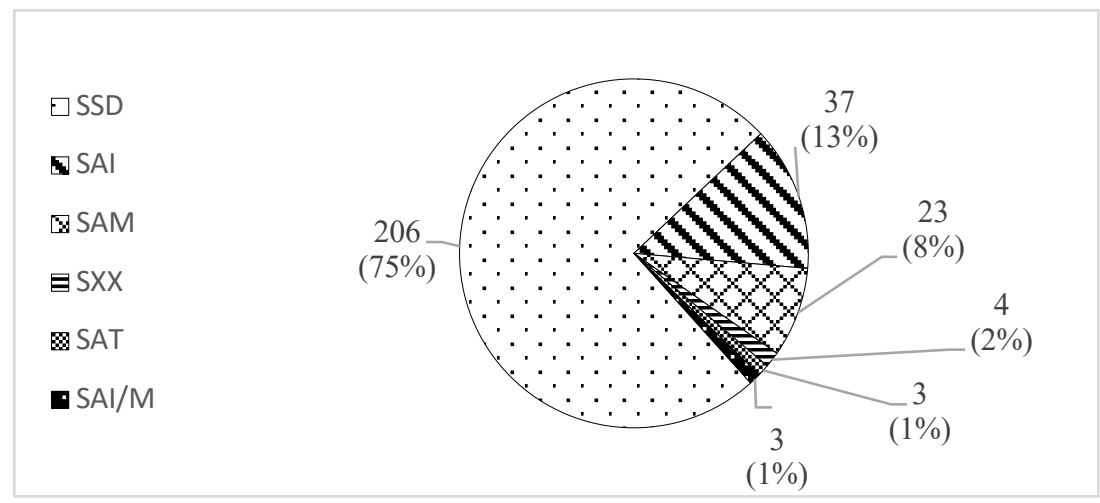

Gráfico 2: Actos formados por un subacto

En el gráfico 2 aparecen los actos formados por un solo subacto. El $75 \%$ de estos actos está formado por un SSD, el $2 \%$ no ha podido establecerse como subacto sustantivo o adyacente y el $23 \%$ restante está compuesto por un subacto adyacente.

Posteriormente, se han aislado los actos formados por dos subactos para analizar su funcionamiento y composición. En el gráfico 3 se muestran los resultados de este análisis:

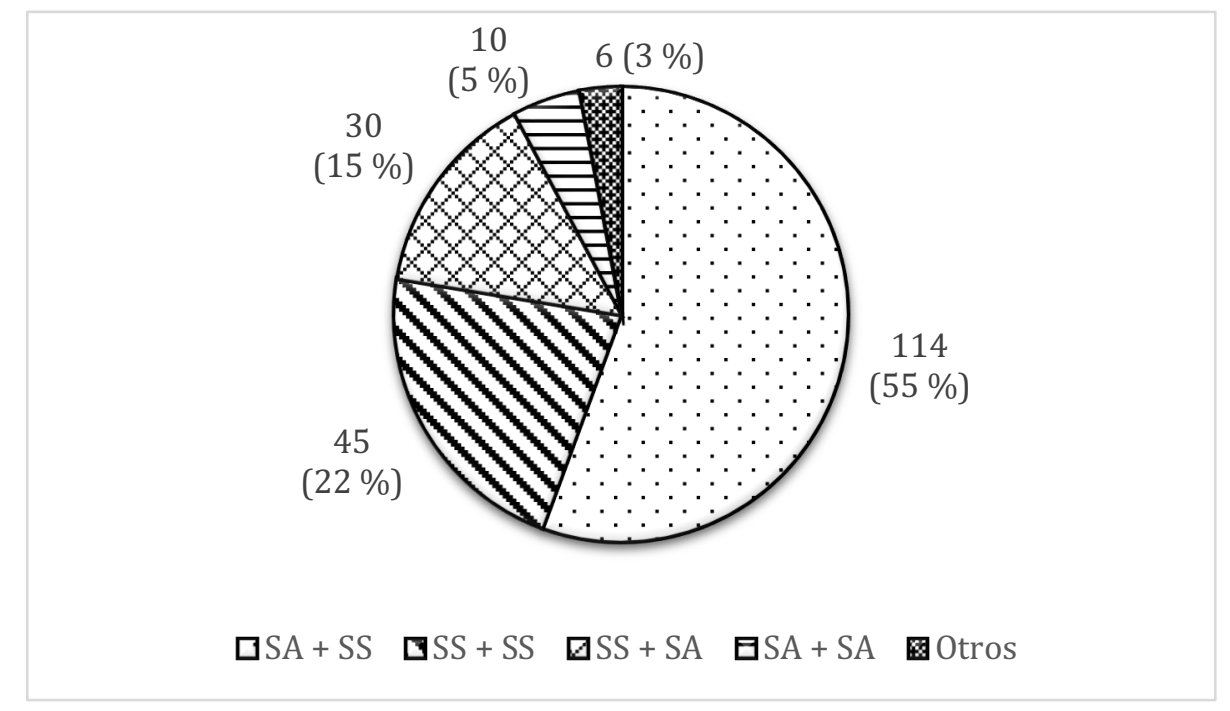

Gráfico 3: Actos formados por dos subactos

En el $55 \%$ de los casos aparece en primer lugar un SA seguido de un SS, mientras que en el $15 \%$ ocurre lo contrario. Este hecho muestra cómo el hablante presenta la información proposicional mediante el uso de información procedimental en primer lugar.

En línea con el tercer objetivo de esta investigación —analizar la ordenación de la información en las intervenciones - se han aislado y estudiado los actos formados por un SSD y un SA, independientemente de su tipo:

\begin{tabular}{|l|l|l|l|}
\hline SAT + SSD & 69 & SSD + SAT & 6 \\
\hline SAM + SSD & 24 & SSD + SAM & 12 \\
\hline SAl + SSD & 14 & SSD + SAl & 10 \\
\hline
\end{tabular}

Tabla 1: Distribución de los subactos SA + SSD 
En la tabla 1 se muestran las seis posibles combinaciones: dos para cada tipo de SA en posición inicial o final. De este modo pueden contrastarse los datos de un mismo tipo de subacto en una y otra posición y los datos de una misma posición ocupada por los diferentes subactos. En todos estos casos es más frecuente expresar la información procedimental en primer lugar. Sin embargo, la diferencia es notablemente superior en el caso de la información textual, ya que es incluso más frecuente encontrar un SAT en posición inicial que un SSD sumando todas sus combinaciones posibles. Ello puede entenderse como la necesidad del hablante de introducir la nueva información y unirla al resto del discurso. El ejemplo (6) es una muestra de ello:

(6) \# [\{SAT ((pues)) SAT\} \{SSD este verano] ((está un pocoo)) SSD \# \# \{SAT es que SAT\} \{SSD no me gusta nada pensar lo que voy a hacer en [verano] SSD\}\#

En el corpus de esta investigación, los elementos que forman parte con más frecuencia los SAT de este corpus son: pero (52), y (33), es que (29), pues (16) y entonces (11).

Respecto a los elementos modalizadores, la información procedimental ocupa el primer lugar en el doble de casos en que lo hace la información proposicional. Esta distribución puede explicarse por su funcionamiento como elementos reactivos a la intervención previa, como ocurre en el ejemplo (7):

(7) B: \# \{SSD [Marcos Blanc] es ((en lo único de lo que)) le defiende SSD\} \{SSSTop en calvo SSSTop\} § \# A: \# \{SAM hombree] SAM $\{$ SSD porque vaya [comparacióon $\rightarrow$ ] SSD \#

Por último, en cuanto a los subactos adyacentes interpersonales, los datos tienen una distribución más equitativa y la información procedimental tiene prácticamente la misma probabilidad de aparecer en posición inicial o final. Se trata de casos como (8):

(8) A: \# \{SSD ¿sabes cuánto cobrann tr- ee seis semanas? [del] SSD $\}$ \#

B: \# \{SSD [menos que vosotros] SSD $\{$ SSAl ¿no? SAl\} \#

Como muestra el ejemplo 8, la forma más empleada por los hablantes en este tipo de subactos es ¿no? Con el objetivo de establecer la validez de estos datos se ha realizado una prueba estadística chi cuadrado, cuyos resultados, bajo un parámetro de normalidad, son los siguientes: las frecuencias obtenidas de aparición son las que muy probablemente se darán si se realiza esta misma investigación con otras conversaciones, $X^{2}(5, N=135)=$ 123,36, $p=<, 0001$. Además, si se observan los residuos estandarizados, se comprueba cómo la combinación SAT + SSD aparece con mucha más frecuencia de lo esperado $(+9,8)^{6}$, lo que indica que, de volver a realizarse esta investigación, volvería a darse esta combinación en un número superior al resto de combinaciones.

En tercer lugar, se han analizado los actos formados por tres subactos. Los cuatro patrones más frecuentes son los que se extraen del gráfico 4:

\footnotetext{
${ }_{6}^{6}$ Un resultado es considerado relevante cuando su residuo estandarizado es superior a 1,96, con una probabilidad del 95 $\%$ de obtener los mismos resultados. 


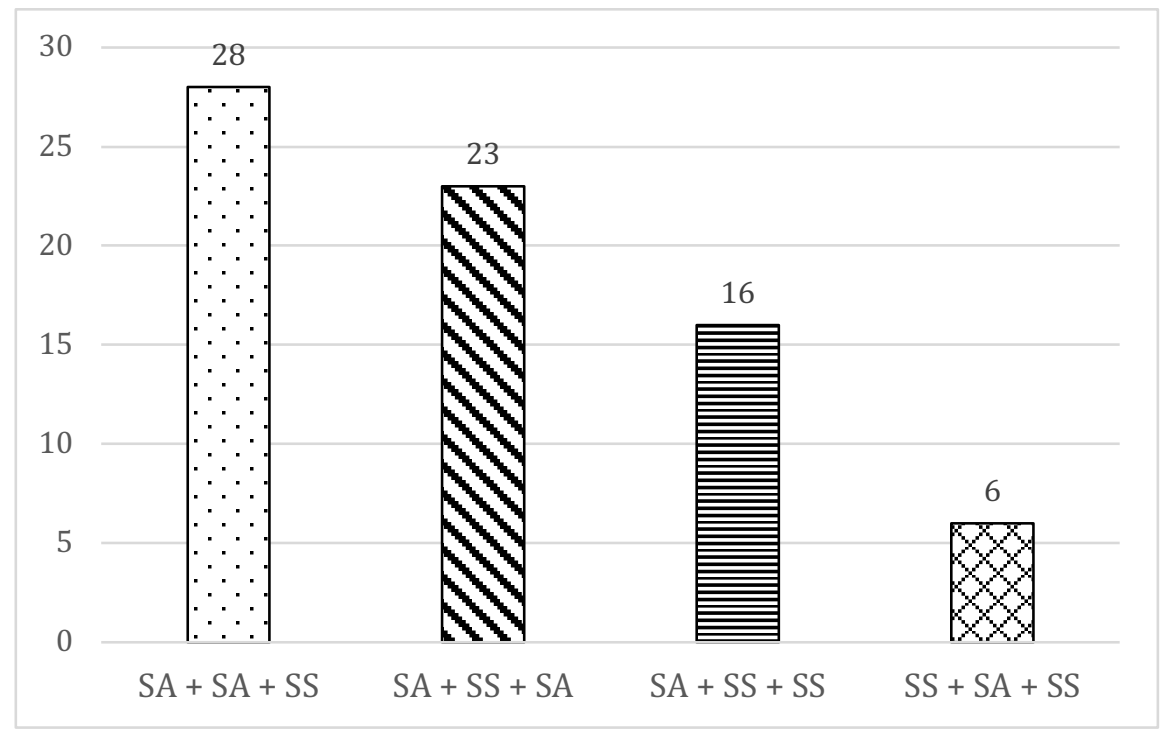

Gráfico 4: Actos formados por tres subactos

Los tres patrones más frecuentes poseen una característica en común: la posición inicial está ocupada por información procedimental. Esta característica, ya analizada en los actos formados por dos subactos, se da de nuevo en los actos formados por tres subactos y su probabilidad de aparición aumenta, ya que el $86 \%$ de este tipo de actos presenta información procedimental en posición inicial. Ejemplo de ello son los dos actos que conforman la intervención de A en (9):

(9) B: \# \{SAT ((que)) SAT\} \{SSD si no podíamos ((haber ido)) a la mascletà SSD\} \# A: \# \{SAT es que SAT\} \{SAT además SAT\} \{SSD supongo que la harán SSD\}\# \#\{SAT bueno SAT\} \{SSD iremos mañana SSD $\{$ SAl ¿no? SAl\} \#

frente a un $14 \%$ de actos con información proposicional en primer lugar, como ocurre en la intervención de A en (10):

(10) A: \# \{SSD [ese] chaval será rico tío de mayor SSD\}/ \{SAT [((es que SAT\} \{SAM estoy segura)) SAM\} ] \#

Por último, tras estudiar los actos simples y los formados por dos y tres subactos, se han agrupado los actos con más de tres subactos, dado que su frecuencia de aparición es bastante inferior al resto. El interés de este análisis se centra en la mayor o menor presencia de SSAA frente a SSSS, independientemente de la posición que ocupen dentro del acto. Los resultados de este estudio se exponen en el gráfico 5: 


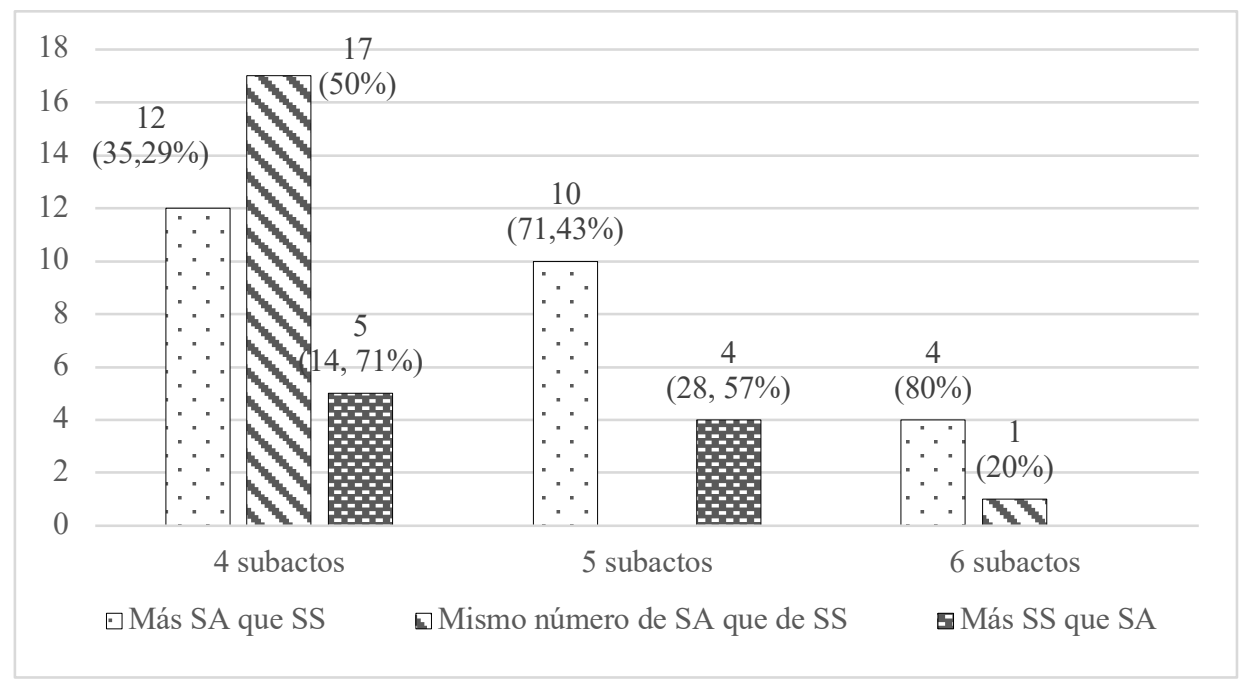

Gráfico 5: Presencia de SA y SS en actos formados por más de tres subactos

Como se extrae de este gráfico, en la mitad de los actos formados por cuatro subactos la presencia entre SSAA y SSSS está equilibrada. En un 35,29\%, aparecen tres SSAA frente a un SS y en el $14,71 \%$ ocurre lo contrario. Es decir, en caso de que haya un mayor número de uno de los tipos de subactos, hay mayor probabilidad de que sea de SSAA.

En el caso de los actos formados por cinco subactos, no es posible que haya el mismo número de SSAA y SSSS y la balanza se inclina por la mayor presencia de SSAA $(71,43$ $\%)$.

Por último, en los actos formados por 6 subactos, no hay ningún caso en el que aparezcan más SSSS que SSAA. En el $80 \%$ hay un mayor número de SSAA y solo en un caso aparece el mismo número de SSSS y de SSAA.

Muestra de estos subactos son los ejemplos (11) y (12), en los que se observa cómo la información procedimental introduce, matiza y delimita el contenido proposicional:

(11) B: \# \{SAM jtela tela! SAM $\{$ SAl oye SAI\} \{SAT ¿y SAT\} \{SSD cuándo vas a sabeer cuándoo cuándo trabajas en la $\rightarrow$ ? SSD \#

(12) B: \# \{SAT ¿y SAT\} \{SSD no les habéis dicho nada? SSD\} / \{SAM no sé SAM\} \{SSS un [escrito o alguna cosa en septiembre] SSS $\}$ \{SAl ¿no? SAl $\#$

El estudio de estos casos señala la relación entre el aumento de información procedimental de distinto tipo con el incremento del número de subactos por acto. Es decir, cuando aparecen actos con un elevado número de subactos, este número está más relacionado con la información procedimental que indica cómo interpretar el contenido proposicional, que con este contenido mismo. En otras palabras, un número elevado de subactos no es indicativo de un mayor contenido proposicional, sino de un mayor número de instrucciones para interpretarlo.

Siguiendo la metodología empleada en este trabajo, se ha analizado la posición inicial en los actos con más de tres subactos: 


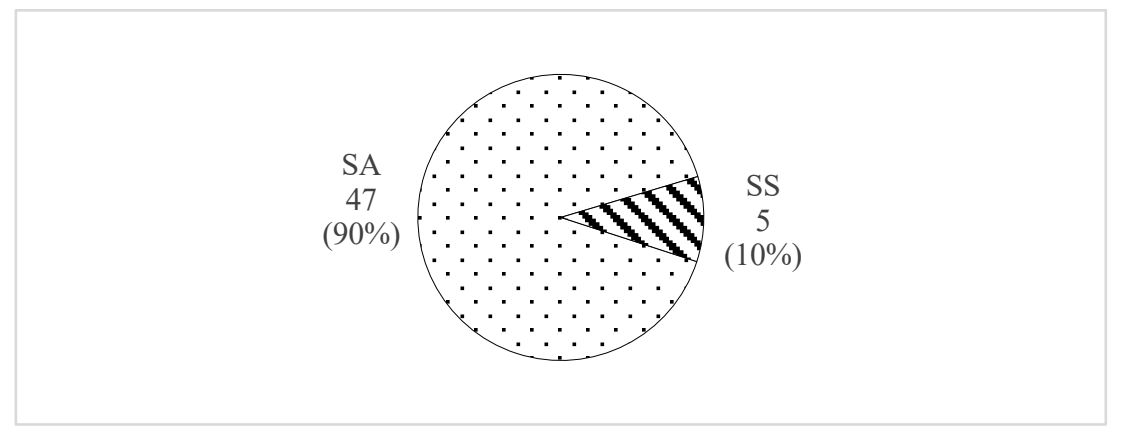

Gráfico 6: Primera posición en actos con más de tres subactos

Como puede observarse en el gráfico 6, en el $91 \%$ de los casos el primer subacto estaba compuesto por información procedimental. Además, los cinco actos iniciados por un SS tienen cuatros subactos y no se ha dado ningún caso con actos de cinco y seis subactos que se inicien con un SS. Estos datos apoyan la percepción de que, a mayor complejidad, mayor necesidad de estructurar el flujo de habla y de añadir información procedimental. De estos 47 casos, el 59,57 \% son textuales; el 21,28 \%, modalizadores; y el 19,15\%, interpersonales.

El tercer objetivo de la investigación consiste en estudiar la estructuración de la información dentro de los actos. Además de los patrones ya expuestos, resulta interesante analizar qué tipo de información ocupa la posición inicial, informativamente más relevante, en los diferentes tipos de subactos:

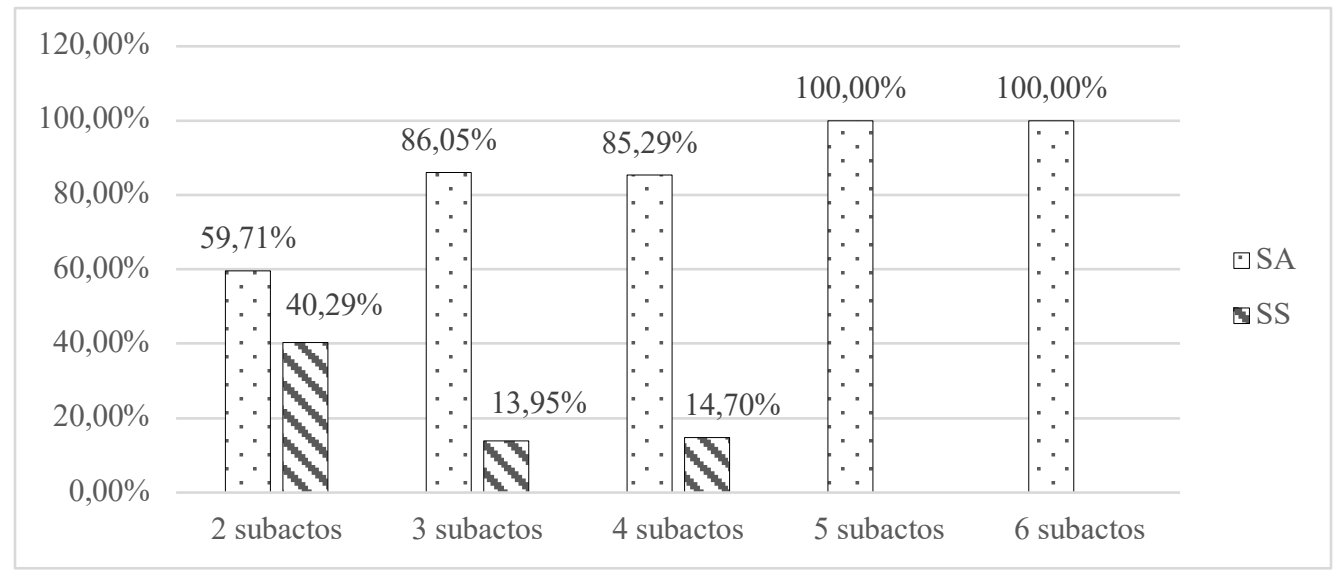

Gráfico 7: Comparación entre subactos en primera posición

El gráfico 7 recoge la evolución que se esboza en los análisis individuales, esto es, a medida que aumenta el número de subactos aumenta la probabilidad de que la posición inicial esté ocupada por un SA. Mientras que en los actos formados por dos subactos hay casi la misma probabilidad de que el primer subacto sea SS que de que sea SA, la frecuencia de SS baja drásticamente en los actos formados por tres subactos, se mantiene en los formados por cuatros subactos y desaparece en los siguientes.

De nuevo, se ha realizado una prueba estadística chi cuadrado para comprobar la probabilidad de volver a encontrar los mismos datos en otras conversaciones. El resultado obtenido es el siguiente: la aparición de un SA o un SS en posición inicial está vinculada con el número de subactos que contiene un acto, $X^{2}(2, N=327)=24,34, p=<, 0001$. En 
este caso, los residuos estandarizados señalan varias cosas: el dato más relacionado con el conjunto es la aparición de SS en primera posición en los actos formados por dos subactos $(+2,49)$. Esto significa que aparece de forma más frecuente de lo que cabría esperar, en relación con el resto de los datos y con una probabilidad de aparición en otras investigaciones del $95 \%{ }^{7}$. Sin embargo, si se mantiene una probabilidad del $90 \%$, se remarca como relevante también la aparición de un SA en primera posición en los actos formados por tres subactos. En definitiva, en relación con los datos de esta investigación, se esperaba una menor frecuencia de aparición de SA en posición inicial $(+1,86)$ y una frecuencia mucho mayor de aparición de SS $(-2,8)$.

\section{CONCLUSIONES}

Esta investigación perseguía tres objetivos. El primero de ellos consistía en estudiar la composición de las intervenciones de la conversación coloquial española. Para ello, se analizaron las unidades que componen estas intervenciones, los actos y los subactos, desde diferentes perspectivas. Primero, clasificando los actos según el número de subactos que los componían; a continuación, observando también las relaciones que se daban entre los diferentes tipos de subactos, su posición y las formas más frecuentes.

En este sentido se puede concluir que las intervenciones están formadas en su mayoría (77\%) por actos simples (44\%) o por actos con dos subactos (33\%). Por un lado, este dato remarca la utilidad de los sistemas de segmentación del discurso para delimitar los fenómenos lingüísticos y facilitar su estudio. Por otro lado, establece la simplicidad estructural de las intervenciones en la conversación coloquial española, favoreciendo así también su análisis.

En esta línea, el segundo objetivo de la investigación trataba de hallar patrones de aparición frecuente característicos de la conversación coloquial española. Durante el análisis de los datos se han destacado los patrones más frecuentes en cada tipo de subacto: SSD (75 $\%)$ en los actos con un subacto, SA + SS (55\%) en los actos con dos subactos y SA + SA + SS (33 \%) en los actos con tres subactos. Como resulta obvio, el aumento del número de subactos aumenta las variables y reduce la frecuencia de un mismo esquema.

El análisis de estos datos nos lleva, a su vez, al tercer objetivo: estudiar la estructuración de la información dentro de los actos. En este punto, se pueden extraer dos conclusiones:

- El aumento del número de subactos dentro de un acto está relacionado con el aumento de información procedimental, es decir, de SSAA que dan instrucciones al interlocutor de cómo interpretar el contenido proposicional de los SSSS.

- Estas instrucciones resultan especialmente necesarias en posición inicial de acto, ya que el hablante tiene la necesidad de introducir la información nueva y unirla con la información ya conocida en el marco de la conversación. Esta necesidad aumenta a medida que aumenta también el número de instrucciones, es decir, a medida que el hablante utiliza un mayor número de SSAA, aumenta la probabilidad de que la posición inicial esté ocupada por un SA.

Este estudio cuantifica y analiza la composición de las intervenciones en la conversación coloquial española. Asimismo, contribuye a los estudios lingüísticos de dos maneras: por

7 Es umbral de aparición es el parámetro empleado habitualmente en las investigaciones científicas. 
un lado, al estudio general de la conversación coloquial, puesto que aporta datos sobre la construcción y las relaciones que se dan en sus unidades más pequeñas; y, por otro lado, a los estudios de fenómenos lingüísticos característicos de este género, ya que aporta un marco de aparición concreto que facilita su delimitación y análisis y muestra algunas de las formas más utilizadas.

\section{REFERENCIAS BIBLIOGRÁFICAS}

Benavent Payá, Elisa (2015): Decir y discurso directo en los relatos de la conversación coloquial, Tesis Doctoral, Universitat de València, Departamento de Filología Española.

Berrendonner, Alain (2011): «Unités syntaxiques \& unités prosodiques», Langue française, 170, 81-93, http://dx.doi.org/10.3917/If.170.0081.

Blanche-benveniste, Claire (1990): Le français parlé. Études gramaticales, París, Éditions du CNRS.

Briz Gómez, Antonio (1996): El español coloquial: situación y uso, Madrid, Arco Libros.

Briz Gómez, Antonio (1998): El español coloquial en la conversación. Esbozo de pragmagramática, Barcelona, Ariel.

Briz, Antonio y Grupo Val.Es.Co. (2003): «Un sistema de unidades para el estudio del lenguaje coloquial», Oralia, 6, 7-61.

Cabedo Nebot, Adrián (2009): Segmentación prosódica en la conversación coloquial: sobre el grupo entonativo como mecanismo demarcativo de unidades mínimas, Tesis Doctoral, Universitat de València, Departamento de Filología Española.

Cabedo Nebot, Adrián y Salvador Pons Bordería, eds. (2013): Corpus Val.Es.Co 2.0 [en línea]: $<$ http://www.valesco.es>.

Cortés Rodríguez, Luis (2002): «Las unidades del discurso oral», Boletín de Lingüística, 17, 7-29.

Cortés Rodríguez, Luis y $\mathrm{M}^{\mathrm{a}}$ Matilde Camacho (2005): Unidades de segmentación y marcadores del discurso: elementos esenciales en el procesamiento discursivo oral, Madrid, Arco Libros.

Degand, Lisbeth y Anne Catherine Simon (2005): "Minimal Discourse Units: Can we define them, and why should we", en Aurnague, Michel et alii, eds., Proceedings of SEM-05. Connectors, discourse framing and discourse structure: from corpus-based and experimental analyses to discourse theories, Biarritz, 14-15 November 2005, 65-74 [en línea]: $<$ http://w3.erss.univ-

tlse2.fr:8080/index.jsp?perso=bras\&subURL=sem0 5/proceedingsfinal/06-Degand-Simon.pdf>.

Degand, Lisbeth y Anne Catherine Simon (2008): "Minimal discourse units in spoken French: Uncovering genre-bound segmentation strategies», en Ramm, Wiebke y Cathrine Fabricius-Hansen, eds., Linearization and Segmentation in Discourse. Multidisciplinary Approaches to Discourse 2008 (MAD 08), 20-23 February 2008, Lysebu, Oslo.

Degand, Lisbeth y Anne Catherine Simon (2009): «On identifying basic discourse units in speech: theoretical and empirical issues», Discours, 4 [en línea]: $<$ https://journals.openedition.org/discours/5852>

Grupo Val.Es.Co (2014): «Las unidades del discurso oral. La propuesta Val.Es.Co. de segmentación de la conversación (coloquial)», Estudios de Lingüística del
Español, 35, 13-73 [en línea]: $<$ http://infoling.org/elies/35/elies35.1.pdf>.

Hidalgo, Antonio y Xose Antonio Padilla (2006): «Bases para el análisis de las unidades menores del discurso oral: los subactos», Oralia, 9, 109-143.

Morel, Mary-Annick y Laurent Danon-Boileau (1998): Grammaire de l'intonation. L'exemple du français oral, París, Ophrys.

Narbona, Antonio (1989a): Las subordinadas adverbiales impropias en español. Bases para su estudio, Málaga, Ágora.

Narbona, Antonio (1989b): Sintaxis española: nuevos y viejos enfoques, Barcelona, Ariel.

Narbona, Antonio (1990): Las subordinadas adverbiales impropias en español (II). Causales y finales, comparativas y consecutivas, condicionales y concesivas, Málaga, Ágora.

Narbona, Antonio (1994): «Hacia una sintaxis del español coloquial», en Actas del Congreso de la Lengua Española, Sevilla 7-10 octubre 1992, Madrid, Instituto Cervantes, 721-740.

Padilla, Xose Antonio (2006): «Las unidades monológicas (intervención, acto y subacto) y el orden de palabras: una guía para su análisis en el discurso hablado», en Casado, Manuel et alii, eds., Análisis del discurso: lengua, cultura, valores, vol. 1, Madrid, Arco Libros, 677-689.

Pascual Aliaga, Elena (2014): Aproximación a la segmentación del subacto en la conversación coloquial española, Trabajo Final de Máster, Universitat de València, Departamento de Filología Española.

Pons Bordería, Salvador, ed. (2014): Discourse Segmentation in Romance Languages, Ámsterdam, John Benjamins.

Pons Bordería, Salvador (2016): "Cómo dividir una conversación en actos y subactos», en Bañón, Antonio Miguel et alii, coords., Oralidad y análisis del discurso: homenaje a Luis Cortés Rodríguez, Almería, Universidad de Almería, 545-566.

Robles, Ferran (2019, en prensa): «Marcadores del discurso y organización informativa del enunciado: los reformuladores explicativos alemanes", Revista de Filología Alemana, 27.

Roulet, Eddy (1991): L'articulation du discours français contemporain, Berna, Peter Lang.

Roulet, Eddy et alii (1985): «Vers une approche modulaire de l'analyse du discours", Cahiers de Linguistique Française, 12, 53-81.

Sloetjes, Han y Peter Wittenburg (2008): «Annotation by category - ELAN and ISO DCR», en Proceedings of the 6th International Conference on Language Resources and Evaluation, Marrakech 2008 [en línea]: <http://www.mpi.nl/publications/escidoc60774/@@popup>. 